\title{
The Inverse Impact-Induced Vibration Problem in Constrained Rotating Systems
}

\author{
Wei-Hsin Gau \\ Department of Mechanical Engineering, Huafan University, Taipei Hsen, Taiwan 223, R.O.C. \\ Ching-Yu Yang \\ Department of Mold and Die Engineering, National Kaobsiung University of Applied Science, \\ Kaobsiung City, Taiwan 807, R.O.C.
}

(Received 11 September 2000; revised 1 July 2001; accepted 5 March 2002)

\begin{abstract}
The analysis presented in this investigation is aimed at the development of a direct scheme to deal with the inverse impact-induced longitudinal vibration problem in constrained rotating systems. An inverse method is developed to estimate the impact conditions, such as the coefficient of restitution, the velocity of the impact mass, and the mass ratio between two bodies. The angular velocity of the rotating body and the properties of the body can also be estimated. The modal expansion method is employed to analyse the problem domain, and then a linear inverse model is constructed to identify the unknowns. The linear least-squares error method is adopted for the linear model and thus the number of iterations can be limited to one and the uniqueness of the solutions can be determined. The use of the procedure described in this paper is demonstrated using a radially-rotating rod subjected to an axial impact. The results presented in this paper show that the scheme is stable and the solution estimated converges to the exact result without the inclusion of the measurement errors.
\end{abstract}

\section{INTRODUCTION}

In this investigation, the solution of the propagation of the impact-induced waves in rotating elastic rods is obtained by using the modal expansion method. The dynamic equations of motion are developed using the principle of virtual work in dynamics. Jump discontinuities in the system velocity vector as the result of an impact are predicted using the generalised impulse momentum equations that involve the coefficient of restitution. The inverse impact-induced vibration problem deals with the determination of the crucial parameters in the analysis of the impact, such as the coefficient of restitution, the velocity of the impact mass, the mass ratio between two bodies, and the generalised impulse. The proposed method is valuable for applications in many design and manufacturing problems, especially when direct measurements of the unknown conditions are not possible. Inverse methods have been implemented in many fields of engineering. Most methods have been used to deal with inverse heat transfer problems, but a few studies have also been conducted on inverse vibration problems. Busby and Trujillo ${ }^{1}$ used modal analysis in conjunction with dynamic programming to investigate the impact force of a structure. They also applied dynamic programming to estimate the magnitude of a time-varying force when the location of the force is known. ${ }^{2}$ Lim and Pilkey ${ }^{3}$ presented a systematic pseudo-inverse solution instead of the dynamic programming solution. Lin, etc. ${ }^{4}$ developed a method for structural analytical model updating. Michaels and $\mathrm{Pao}^{5-6}$ determined the inverse solutions via a deconvolution method for multiple Green's functions for application to experimental data. Despite all the efforts which have been made in the past, the iterative process can not be avoided and that the inverse problem must be solved in a nonlinear domain. Therefore, it is necessary to find a method that can be used to estimate inverse vibration problems in a linear domain.

In the present research, the objective is to develop a direct scheme to deal with inverse impact-induced vibration problems. ${ }^{7-8}$ The purpose of this paper is to propose a methodology that can be used to directly estimate the impact conditions, such as the coefficient of restitution, the velocity of the impact mass, the mass ratio between two bodies, and the generalised impulse. In the method proposed, a set of function forms with the unknown variables are first defined in the equation. Then the temporal coordinate is substituted into the equation created in the first step. After that, the equation becomes a set of equations with the unknown variables. As a result, this inverse model can be used to find a solution of the problem through the linear least-squares error method.

\section{DYNAMIC EQUATIONS}

In this section, the direct method of formulating the equations of vibration for the radial-rotation of the rod shown in Fig. 1 is developed. The rod is assumed to rotate with a constant angular velocity and the coupling between the transverse and the axial deformation is neglected. The global position of an arbitrary point on the rod can be written as

$$
\mathbf{r}=\mathbf{A u},
$$

where $\mathbf{A}$ is the planar rotation matrix, and $\mathbf{u}$ is the local position vector of the arbitrary point on the rod. The matrix $\mathbf{A}$ and the vector $\mathbf{u}$ are given by

$$
\begin{gathered}
\mathbf{A}=\left[\begin{array}{cc}
\cos \theta & -\sin \theta \\
\sin \theta & \cos \theta
\end{array}\right] ; \\
\mathbf{u}=\mathbf{u}_{0}+\mathbf{u}_{f},
\end{gathered}
$$

International Journal of Acoustics and Vibration, Vol. 7, No. 3, 2002 (pp 159-164) 